\title{
ON $\mu$-ESSENTIAL AND $\mu-M$-SINGULAR MODULES
}

\author{
A. Çiğdem ÖZCAN \\ Hacettepe University \\ Department of Mathematics \\ 06800 Beytepe Ankara, Turkey \\ E-mail: ozcan@hacettepe.edu.tr
}

\begin{abstract}
As a generalization of essential submodules Zhou defines a $\mu$-essential submodule provided it has a non-zero intersection with any non-zero submodule in $\mu$ for any class $\mu$. Let $M$ be a module. In this article we study $\delta$-essential submodules as a dual of $\delta$-small submodules of Zhou where $\delta=\{N \in \sigma[M]: \operatorname{Rej}(N, \mathcal{M})=0\}$ and $\mathcal{M}=\{N \in \sigma[M]: N \ll$ $\widehat{N}\}$, and also define $\mu$ - $M$-singular modules as modules $N \in \sigma[M]$ such that $N \cong K / L$ for some $K \in \sigma[M]$ and $L$ is $\mu$-essential in $K$. By $\mathcal{M}-M$-singular modules and $\delta-M-$ singular modules a characterization of $\mathrm{GCO}-$ modules, and by $\mathcal{F C}-M$-singular modules where $\mathcal{F C}$ is the class of finitely cogenerated modules, a characterization of semisimple Artinian rings are given.
\end{abstract}

Keywords: essential submodule, singular module

\section{Preliminaries}

Let $M$ be a module, $N \in \sigma[M]$ and $\mu$ a class of modules in $\sigma[M]$ which is closed under isomorphisms and submodules. Following Zhou ${ }^{16}$ we call a submodule $N$ a $\mu-$ essential submodule of $K \in \sigma[M]$ if for any nonzero $\mu$-submodule $X$ in $K, N \cap X \neq 0$, denoted by $N \leq_{\mu e} K$. In this article after studying some properties of $\mu$-essential submodules we consider $\delta$-essential submodules as a dual of $\delta$-small submodules of Zhou by denoting the class $\delta=\left\{N \in \sigma[M]: \operatorname{Rej}(N, \mathcal{M}):=\bar{Z}_{M}(N)=0\right\}$ where $\mathcal{M}=\{N \in \sigma[M]: N \ll \widehat{N}\}$ and $\bar{Z}_{M}($.$) is defined by Talebi and Vanaja as a dual$ of the singular submodule $Z_{M}($.$) . If \mathcal{F}=\left\{F \in \sigma[M]: \forall 0 \neq K \subseteq F, \bar{Z}_{M}(K) \neq K\right\}$, then it is known that $\mathcal{M} \subseteq \delta \subseteq \mathcal{F}$. We prove a result on when an $\mathcal{F}$-essential submodule is $\delta$-essential and a $\delta$-essential submodule is $\mathcal{M}$-essential. Also we prove that $\operatorname{Tr}(\mathcal{S} \cap \mathcal{M}, N)=\operatorname{Tr}(\mathcal{S} \cap \delta, N)=\operatorname{Tr}(\mathcal{S} \cap \mathcal{F}, N)$ where $\mathcal{S}$ is the class of simple modules in $\sigma[M]$ and $\operatorname{Tr}$ is used for the trace.

In the last section we define $\mu-M$-singular modules $N \in \sigma[M]$ for a module $M$. $N$ is called $\mu$-M-singular module if $N \cong K / L$ for some $K \in \sigma[M]$ and $L \leq_{\mu e} K$. It is proved that $M$ is a GCO-module (i.e. every simple $M$-singular module is injective in $\sigma[M])$ if and only if for every $\mathcal{M}-M$-singular module $N$ in $\sigma[M], \bar{Z}_{M}(N)=N$ if and only if for every $\delta$-M-singular module $N$ in $\sigma[M], \bar{Z}_{M}(N)=N$. When we consider the class of all finitely cogenerated modules $\mathcal{F C}$ we prove that every finitely cogenerated $R$-module is projective if and only if for every $\mathcal{F C}-R$-singular 
$R$-module $N, \operatorname{Rej}(N, \mathcal{F} C)=N$ if and only if $R$ is semisimple Artinian.

Let $R$ be a ring with identity. All modules we consider are unitary right $R$ modules and we denote the category of all such modules by Mod- $R$. Let $M$ be an $R$-module. The $R$-injective hull of $M$ is denoted by $\mathrm{E}(M)$, and the $M$-injective hull of $N$ in the category of $\sigma[M]$ is denoted by $\widehat{N}$. For the definition of $\sigma[M]$ and $\widehat{N}$ see. ${ }^{15}$

Let $\mu$ be a class of modules. For any module $N$, the trace of $\mu$ in $N$ is denoted by $\operatorname{Tr}(\mu, N)=\Sigma\{\operatorname{Imf}: f \in \operatorname{Hom}(C, N), C \in \mu\}$. Dually the reject of $\mu$ in $N$ is denoted by $\operatorname{Rej}(N, \mu)=\bigcap\{k \operatorname{kerg}: g \in \operatorname{Hom}(N, C), C \in \mu\}$.

Let $N$ be a submodule of $M(N \leq M)$. The notations $N \ll M, N \leq_{e} M$ and $N \leq_{d} M$ is used for a small submodule, an essential submodule and a direct summand of $M$, respectively. $\operatorname{Soc}(M)$ will denote the socle of $M$. A module $N \in$ $\sigma[M]$ is said to be $M$-small (or small in $\sigma[M]$ ) if $N \cong K \ll L$ for $K, L \in \sigma[M]$. Then an $R$-module $N \in \sigma[M]$ is $M$-small if and only if $N \ll \widehat{N}$.

Dually, a module $N \in \sigma[M]$ is called $M$-singular (or singular in $\sigma[M]$ ) if $N \cong$ $L / K$ for an $L \in \sigma[M]$ and $K \leq_{e} L$. Every module $N \in \sigma[M]$ contains a largest $M-$ singular submodule which is denoted by $\mathrm{Z}_{M}(N)$. Then $\mathrm{Z}_{M}(N)=\operatorname{Tr}(\mathcal{U}, N)$ where $\mathcal{U}$ denotes the class of all $M$-singular modules $\left(\operatorname{see}^{15}\right)$.

Simple modules in $\sigma[M]$ split into four disjoint classes by combining the exclusive choices [ $M$-injective or $M$-small] and [ $M$-projective or $M$-singular]. Also note that if a module $N$ in $\sigma[M]$ is $M$-singular and projective in $\sigma[M]$, then it is zero.

Let $N \subseteq K \in \sigma[M] . N$ is called $\delta-M-$ small in $K$ if, whenever $N+X=K$ with $K / X$ is $M$-singular, we have $X=K\left(\mathrm{see}^{7}\right)$. Zhou ${ }^{17}$ studies $\delta-R$-small submodules in Mod- $R$. By [17, Lemma 1.2], in the definition of $\delta-R$-small submodule, $K / X$ can be taken Goldie torsion, i.e. $K / X$ can be a member of the torsion class of the Goldie torsion theory in Mod- $R$.

In this paper $\mu$ will be a class in $\sigma[M]$ which is closed under isomorphisms and submodules, unless otherwise stated. Any member of $\mu$ we shall call a $\mu$-module. In this article we denote the following classes:

$$
\begin{aligned}
& \mathcal{S}=\{N \in \sigma[M]: N \text { is simple }\}, \\
& \mathcal{M}=\{N \in \sigma[M]: N \text { is } M \text {-small }\} \\
& \delta=\left\{N \in \sigma[M]: \bar{Z}_{M}(N)=0\right\}, \\
& \mathcal{F}=\left\{F \in \sigma[M]: \forall 0 \neq K \subseteq F, \bar{Z}_{M}(K) \neq K\right\} \\
& \mu M-\operatorname{Sing}=\{N \in \sigma[M]: N \text { is } \mu \text { - } M \text {-singular }\} \\
& \mathcal{F C}=\{N \in \sigma[M]: N \text { is finitely cogenerated }\}
\end{aligned}
$$

Definition 1.1. Let $N \in \sigma[M]$. Following Zhou ${ }^{16} N$ is called a $\mu$-essential submodule of $K \in \sigma[M]$ if for any nonzero $\mu$-module $X$ in $K, N \cap X \neq 0$. It is denoted by $N \leq_{\mu e} K$.

Clearly every essential submodule is $\mu$-essential. But the converse is not true in general. 
Example 1.1. Let $\mu$ be the class of simple modules and zero modules in Mod- $R$. Then a submodule $N$ of a module $M$ is $\mu$-essential if and only if $N$ contained the socle of $M$ but this is not enough to make $N$ essential. For example in the $\mathbb{Z}$-module $\mathbb{Z} \oplus \mathbb{Z}_{p}$, where $p$ is a prime, $0 \oplus \mathbb{Z}_{p}$ is $\mu$-essential but not essential.

Example 1.2. Consider the class of $M$-small modules $\mathcal{M}$ in $\sigma[M]$. Let $N$ be an injective module in $\sigma[M]$ with $0 \neq \operatorname{Rad}(N) \underline{L}_{e} N$ (for example let $N=U \oplus V$ where $U$ is injective with essential radical and $V$ is injective simple module.) Let $X$ be a non-zero $M$-small submodule of $N$. Then $X \ll N$ so $X=X \cap \operatorname{Rad}(N) \neq 0$. Thus $\operatorname{Rad}(N)$ is $\mathcal{M}$-essential but not essential in $N$.

The following lemma is clear from definitions.

Lemma 1.1. Let $K \in \sigma[M]$. If every nonzero submodule of $K$ contains a nonzero $\mu$-module, then for any submodule $N$ of $K, N \leq_{e} K$ if and only if $N \leq_{\mu e} K$.

Corollary 1.1. Let $N \leq K \in \sigma[M]$. If $N \leq_{\mu e} K$ and $K$ is a $\mu$-module, then $N \leq_{e} K$.

Now we list the properties of $\mu$-essential submodules. We omit the proofs because they are similar to those for essential submodules (see, for example ${ }^{2}$ ).

Lemma 1.2. Let $M$ be a module.

a) Let $N \leq L \leq K \in \sigma[M]$. Then $N \leq_{\mu e} K$ if and only if $N \leq_{\mu e} L \leq_{\mu e} K$.

b) If $K_{1} \leq_{\mu e} L_{1}, K_{2} \leq_{\mu e} L_{2}$, then $K_{1} \cap K_{2} \leq_{\mu e} L_{1} \cap L_{2}$ for $L_{1}, L_{2} \in \sigma[M]$.

c) Let $N, L \in \sigma[M]$. If $f: N \rightarrow L$ is a homomorphism and $K \leq_{\mu e} L$, then $f^{-1}(K) \leq_{\mu e} N$.

d) If $N / L \leq_{\mu e} K / L$, then $N \leq_{\mu e} K$.

e) Let $N \in \sigma[M],\left\{K_{i}\right\}$ an independent family of submodules of $N$ and if $K_{i} \leq_{\mu e}$ $L_{i} \leq N$ for all $i \in I$, then $\oplus_{i \in I} K_{i} \leq_{\mu e} \oplus_{i \in I} L_{i}$.

Example 1.3. In Lemma 1.2(e), $\left\{L_{i}\right\}$ need not be an independent family. For example, let $\mu$ be the class of simple modules and zero modules and put $K_{1}=$ $0 \oplus \mathbb{Z}_{p} \leq \mathbb{Z} \oplus \mathbb{Z}_{p}=L_{1}$ and $K_{2}=L_{2}=\mathbb{Z} \oplus \overline{0} \leq L_{1}$. Then $K_{1} \leq_{\mu e} L_{1}, K_{2} \leq_{\mu e} L_{2}$ and $K_{1} \cap K_{2}=0$ but $L_{1} \cap L_{2} \neq 0$.

\section{2. $\delta$-essential Submodules Where $\delta=\left\{N \in \sigma[M] \mid \bar{Z}_{M}(N)=0\right\}$}

Talebi and Vanaja ${ }^{14}$ define $\bar{Z}_{M}(N)$ as a dual of $\mathrm{Z}_{M}(N)$ as follows:

$$
\bar{Z}_{M}(N)=\operatorname{Rej}(N, \mathcal{M})=\cap\{\operatorname{ker} g \mid g \in \operatorname{Hom}(N, L), L \in \mathcal{M}\}
$$

where $N \in \sigma[M]$. They call $N$ an $M$-cosingular (non-M-cosingular) module if $\bar{Z}_{M}(N)=0\left(\bar{Z}_{M}(N)=N\right)$. If $N$ is $M$-small, then $N$ is $M$-cosingular. The class of all $M$-cosingular modules is closed under submodules, direct sums and direct products [14, Corollary 2.2]. Note that $\bar{Z}_{M}^{2}(N)=\bar{Z}_{M}\left(\bar{Z}_{M}(N)\right)$. Talebi and Vanaja 
study the torsion theory cogenerated by $M$-small modules, $\tau=(\mathcal{T}, \mathcal{F})$ where

$$
\begin{aligned}
& \mathcal{T}=\left\{T \in \sigma[M] \mid \bar{Z}_{M}(T)=T\right\}, \\
& \mathcal{F}=\left\{F \in \sigma[M] \mid \forall 0 \neq K \leq F, \bar{Z}_{M}(K) \neq K\right\}
\end{aligned}
$$

This torsion theory is also studied by Özcan and Harmanc1. ${ }^{9}$ This is a dual of the Goldie torsion theory and not necessarily hereditary. Also $\mathcal{M} \subseteq \delta \subseteq \mathcal{F}$. Now we investigate the relationship between $\mathcal{M}$-essential, $\delta$-essential and $\mathcal{F}$-essential submodules by inspired [17, Lemma 1.2]. First we note that the following two theorems which are characterize the torsion free class $\mathcal{F}$.

Theorem 2.1. [9, Theorem 15] Let $M$ be a module and assume that $M$ has a projective cover in $\sigma[M]$. If $\bar{Z}_{M}(M)=M$, then $\mathcal{M}=\delta=\mathcal{F}$.

Let $N$ and $L$ be submodules of a module $M . N$ is called a supplement of $L$ (in $M)$ if $N+L=M$ and $N \cap L \ll N . M$ is called amply supplemented if, for all submodules $N$ and $L$ of $M$ with $N+L=M, N$ contains a supplement of $L$ in $M$.

Theorem 2.2. [14, Theorem 3.6] Let $M$ be a module such that every injective module in $\sigma[M]$ is amply supplemented. Then $\mathcal{F}$ is closed under factor modules and $\mathcal{F}=\left\{N \in \sigma[M] \mid \bar{Z}_{M}^{2}(N)=0\right\}$.

For shortness we denote

(A) $M$ has a projective cover and $\bar{Z}_{M}(M)=M$.

(B) Every injective module in $\sigma[M]$ is amply supplemented.

Proposition 2.1. Consider the following conditions for $K \leq N \in \sigma[M]$.

a) $K \leq_{\mathcal{F} e} N$.

b) $K \leq_{\delta e} N$.

c) $K \leq_{\mathcal{M} e} N$.

Then $(a) \Rightarrow(b) \Rightarrow(c)$. If $M$ has (B), then $(b) \Rightarrow(a)$. If $M$ has (A), then $(c) \Rightarrow(a)$.

Proof. $(a) \Rightarrow(b) \Rightarrow(c)$ They are clear.

$(b) \Rightarrow(a)$ Let $X \leq N$ with $X \cap K=0$ and $X \in \mathcal{F}$. Then $\bar{Z}_{M}^{2}(X)=\bar{Z}_{M}\left(\bar{Z}_{M}(X)\right)=0$ by Theorem 2.2. Since $\bar{Z}_{M}(X) \cap K=0, \bar{Z}_{M}(X)=0$ by (b). Again by (b), $X=0$. $(c) \Rightarrow(a)$ It is clear by Theorem 2.1 .

Let $M$ be a module. Define

$$
\operatorname{Soc}_{M}(N)=\operatorname{Tr}(\mathcal{S} \cap \mathcal{M}, N)
$$

for any module $N \in \sigma[M]$. Then $\operatorname{Soc}_{M}(N) \leq \operatorname{Soc}(N)$. Clearly if $\operatorname{Soc}_{M}(N) \leq_{e} N$, then $\operatorname{Soc}_{M}(N)=\operatorname{Soc}(N)$. The following lemma shows that in the definition of $\operatorname{Soc}_{M}(N)$ we can take $\mathcal{F}$-modules or $\delta$-modules instead of $\mathcal{M}$-modules. That is

$$
\operatorname{Soc}_{M}(N)=\operatorname{Tr}(\mathcal{S} \cap \delta, N)=\operatorname{Tr}(\mathcal{S} \cap \mathcal{F}, N) .
$$


Lemma 2.1. Let $M$ be a module. Any simple $\mathcal{F}$-module in $\sigma[M]$ is $M$-small.

Proof. Let $X$ be a simple $\mathcal{F}$-module in $\sigma[M]$. If $\bar{Z}_{M}(X)=X$, then $X \in \mathcal{T} \cap \mathcal{F}=0$, a contradiction.Then $\bar{Z}_{M}(X)=0$. If $X$ is $M$-injective, then $\bar{Z}_{M}(X)=X$. For, let $L$ be a submodule of $X$ such that $X / L$ is $M$-small. If $L=0$, then $X$ is $M$-small, a contradiction. Hence $L=X$, that is $\bar{Z}_{M}(X)=X$. This contradiction implies that $X$ is $M$-small.

The following proposition can be seen by [16, Proposition 3], but we give the proof for completeness.

Proposition 2.2. Let $N \in \sigma[M]$. $\operatorname{Soc}_{M}(N)$ is the intersection of all its $\mathcal{F}$-essential submodules of $N$.

Proof. Let $S$ be a simple $M$-small submodule of $N$ and $K$ be an $\mathcal{F}$-essential submodule of $N$, then $S \cap K \neq 0$. Therefore $S \leq K$. It follows that the intersection of all $\mathcal{F}$-essential submodules contains all simple $M$-small submodules and hence it contains their sum. Thus $\operatorname{Soc}_{M}(N)$ is contained in the intersection of all $\mathcal{F}$-essential submodules of $N$.

If $N=\operatorname{Soc}_{M}(N)$, then the proof is completed. Suppose that $N \neq \operatorname{Soc}_{M}(N)$. Let $n \in N-\operatorname{Soc}_{M}(N)$. Then there exists a submodule $K$ maximal with respect to $K \supseteq \operatorname{Soc}_{M}(N)$ and $n \notin K$. If we can show that $K \leq_{\mathcal{F} e} N$, then $n$ lies outside an $\mathcal{F}$-essential submodule, and so $\operatorname{Soc}_{M}(N)$ is the intersection of all $\mathcal{F}$-essential submodules of $N$.

Suppose that $L \cap K=0$ for some nonzero submodule $L$ of $N$ with $L \in \mathcal{F}$. Consider the natural epimorphism $\pi: N \rightarrow N / K$. Then $L \cong \pi(L) \leq N / K$. Since $K$ is maximal with respect to $K \supseteq \operatorname{Soc}_{M}(N)$ and $n \notin K, N / K$ has a minimal submodule contained in every nonzero submodule. Also since $L \in \mathcal{F}$, then $L \cap$ $\operatorname{Soc}_{M}(N) \neq 0$ by Lemma 2.1. But $L \cap \operatorname{Soc}_{M}(N) \leq L \cap K=0$, a contradiction.

Hence intersections of $\mathcal{M}$-essential, $\delta$-essential and $\mathcal{F}$-essential submodules are equal.

$\operatorname{Tr}(\mathcal{M}, N)$ is investigated in ${ }^{7}$ and denoted by $Z_{M}^{*}(N)$. Then it can be seen that

$$
\operatorname{Soc}\left(Z_{M}^{*}(N)\right)=\operatorname{Soc}_{M}(N) .
$$

There are some examples of modules $M$ such that $\operatorname{Soc}_{M}(N) \neq 0, \operatorname{Soc}_{M}(N) \neq$ $\operatorname{Soc}(N)$ and $\operatorname{Soc}_{M}(N)=\operatorname{Soc}(N)$.

Example 2.1. 1) If $M$ is a cosemisimple module (i.e. every simple module is $M-$ injective) and $N \in \sigma[M]$, then $\operatorname{Soc}_{M}(N)=0$, because $\operatorname{Soc}(N)=\operatorname{Soc}_{M}(N) \oplus T$ where $T$ is a direct sum of simple $M$-injective submodules of $N$.

2) If $R$ is a small ring (for example a commutative integral domain) then every finitely generated $R$-module is small. ${ }^{12}$ This implies that $\operatorname{Soc}_{R}(N)=\operatorname{Soc}(N)$ for 
every $R$-module $N$.

3) Let $R$ be the ring $\left[\begin{array}{ll}F & F \\ 0 & F\end{array}\right]$ where $F$ is a field. Then $Z^{*}\left(R_{R}\right)=\operatorname{Soc}\left(R_{R}\right)$ by [6, Example 11]. This imples that $\operatorname{Soc}\left(Z^{*}\left(R_{R}\right)\right)=\operatorname{Soc}_{R}\left(R_{R}\right)=\operatorname{Soc}\left(R_{R}\right)$. Since $\operatorname{Soc}\left(R_{R}\right) \leq_{e} R_{R}$, every $\delta$-essential right ideal is essential.

$4)^{4}$ Let $Q$ be a local quasi-Frobenius ring and $J=J(Q)$ (the Jacobson radical of $Q), S=\operatorname{Soc}\left(Q_{Q}\right)=\operatorname{Soc}\left({ }_{Q} Q\right)$. Then $W=\left[\begin{array}{cc}Q & Q / S \\ J & Q / S\end{array}\right]$ is a well-defined ring by the usual matrix addition, equality and the following multiplication

$$
\left[\begin{array}{ll}
u & v+S \\
j & w+S
\end{array}\right]\left[\begin{array}{ll}
x & y+S \\
k & z+S
\end{array}\right]=\left[\begin{array}{ll}
u x+v k & u y+v z+S \\
j x+w k & j y+w z+S
\end{array}\right]
$$

where $u, v, w, x, y, z \in Q$ and $j, k \in J . W$ is a right and left Artinian ring. $J(W)=$

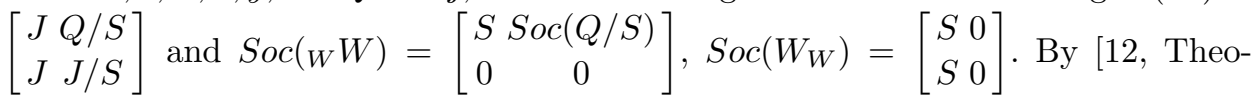
rem 3] or [8, Proposition 2.8], it can be shown that $Z^{*}\left(W_{W}\right)=l_{W}\left(\operatorname{Soc}\left({ }_{W} W\right)\right)=$ $\left[\begin{array}{ll}J & Q / S \\ J & Q / S\end{array}\right]$ where $l_{W}($.$) is the left annihilator over W$. Since $S \leq J$, then $\operatorname{Soc}\left(W_{W}\right) \leq$ $Z^{*}\left(W_{W}\right)$. This implies that $\operatorname{Soc}_{W}\left(W_{W}\right)=\operatorname{Soc}\left(W_{W}\right)$.

$5)^{5}$ Let $R=F[x ; \sigma]$ be the twisted polynomial rings where $F$ is a field of characteristic $p>0$ and $\sigma: F \rightarrow F$ is the endomorphism given by $\sigma(a)=a^{p}(a \in F)$. The ring $R$ consists of all polynomials $a_{0}+x a_{1}+x^{2} a_{2}+\ldots+x^{n} a_{n}$ where $n$ is a non-negative integer, $a_{i} \in F(0 \leq i \leq n)$, multiplication is given by the relation $a x=x \sigma(a)(a \in F)$ Note that $R$ is a principal right ideal domain [5, p.597]. Let $A$ denote the ideal $x R$ of $R$. Clearly $A$ is a maximal right ideal of $R$ and the $R$-module $R / A$ is not injective because $R / A \neq(R / A) x$ (see [13, Proposition 2.6]). In [5, Proposition 9], it is given an example of a field $F$ such that the $R$-module $R / s R$ is injective for all $s \in R-x R$. Thus some simple $R$-modules are injective and some are not. In particular, for the principal right ideal domain $R, Z^{*}\left(M_{1}\right)=M_{1}$ and $Z^{*}\left(M_{2}\right)=0$ for some simple $R$-modules $M_{1}$ and $M_{2}$. In this case, $Z^{*}\left(M_{1} \oplus M_{2}\right)=M_{1} \oplus 0 \neq 0, M_{1} \oplus M_{2}$ (see [8, p.4918]). Hence $\operatorname{Soc}_{R}\left(M_{1} \oplus M_{2}\right)=\operatorname{Soc}\left(Z^{*}\left(M_{1} \oplus M_{2}\right)\right)=M_{1} \oplus 0 \neq \operatorname{Soc}\left(M_{1} \oplus M_{2}\right)$.

\section{3. $\mu-M-$ Singular Modules}

Definition 3.1. Let $M$ be a module and $N \in \sigma[M] . N$ is called $\mu-M$-singular if $N \cong K / L$ for some $K \in \sigma[M]$ and $L \leq_{\mu e} K$. In case $M=R$, we use $\mu$-singular.

The class of $\mu-M$-singular modules is closed under submodules, homomorphic images, direct sums and isomorphisms. 
Hence every module $N \in \sigma[M]$ contains a largest $\mu-M$-singular submodule which we denote by $\mathrm{Z}_{\mu M}(N)=\operatorname{Tr}(\mu M$-Sing, $N)$ where $\mu M$-Sing is the class of all $\mu-M$-singular modules. Then $\mathrm{Z}_{M}(N) \leq \mathrm{Z}_{\mu M}(N)$. If $N \in \sigma[M]$ is $\mu-M$-singular (i.e. $\mathrm{Z}_{\mu M}(N)=N$ ) and a $\mu$-module, then $N$ is $M$-singular. For, let $N \in \mu$ and $N \cong K / L$ where $K \in \sigma[M], L \leq_{\mu e} K$. We claim that $L \leq_{e} K$. Let $0 \neq X \leq K$ and assume that $L \cap X=0$. Then $X \cong(L \oplus X) / L \leq K / L$ and so $X \in \mu$. Since $L \leq_{\mu e} K$ we have a contradiction. This proves that $N$ is $M$-singular.

If $\mathrm{Z}_{\mu M}(N)=0$, then $N$ is called non- $\mu$-singular in $\sigma[M]$ or non- $\mu-M-$ singular.

Proposition 3.1. Let $N$ be a $\mu-M$-singular module and $f \in \operatorname{Hom}_{R}(M, N)$.

(1) If $M$ is quasi-projective and $f(M)$ is finitely generated, then kerf $\leq_{\mu e} M$.

(2) If $M$ is projective in $\sigma[M]$, then kerf $\leq_{\mu e} M$.

Proof. (1) We may assume $f(M) \cong L / K$ where $L \in \sigma[M]$ is finitely generated and $K \leq_{\mu e} L$. Since $L \in \sigma[M]$ and $L$ is finitely generated, then $M$ is $L$-projective. Hence there exists a homomorphism $g: M \rightarrow L$ such that $\pi g=f$ where $\pi$ is the natural epimorphism $L \rightarrow L / K$. Then $\operatorname{ker} f=g^{-1}(K) \leq_{\mu e} M$ by Lemma 1.2.

(2) By the proof of (1).

Proposition 3.2. Let $P$ be a projective $R$-module and $X \leq P$. Then $P / X$ is $\mu$-singular if and only if $X \leq_{\mu e} P$.

Proof. If $I \leq R_{R}$ and $R / I$ is $\mu$-singular, then $I \leq_{\mu e} R$ by Proposition 3.1. Now let $P / X$ be $\mu$-singular and assume $X \mathbb{Z}_{\mu e} P$. Let $F$ be a free module such that $F=P \oplus P^{\prime}, P^{\prime} \leq F$. Then $F /\left(X \oplus P^{\prime}\right) \cong P / X$ is $\mu$-singular and $X \oplus P^{\prime} \mathbb{Z}_{\mu e} F$. So we may assume without loss of generality $P$ is free, i.e. $P=\oplus R_{\lambda}$, each $R_{\lambda}$ is a copy of $R$. Take $R_{\lambda}$. Then $R_{\lambda} /\left(R_{\lambda} \cap X\right) \cong\left(R_{\lambda}+X\right) / X \hookrightarrow P / X$ is $\mu$-singular. So $R_{\lambda} \cap X \leq_{\mu e} R_{\lambda}$. This implies that $\oplus R_{\lambda} \cap X \leq_{\mu e} \oplus R_{\lambda}=P$, i.e. $X \leq_{\mu e} P$.

From the properties of $\mu$-singular modules and the above propositions the following can be seen easily.

Proposition 3.3. For an $R$-module $N$ the following are equivalent.

a) $N$ is $\mu$-singular (in Mod-R).

b) $N \cong F / K$ with $F$ a projective (free) $R$-module and $K \leq_{\mu e} F$.

c) For every $n \in N$, the right annihilator $r(n)$ is $\mu$-essential in $R$.

Recall that a submodule $N$ of a module $M$ is said to be closed in $M$ if $N$ has no proper essential extension in $M$, denote $N \leq_{c} M$.

Lemma 3.1. Let $M$ be a module and $N \in \sigma[M]$. If $Z_{\mu M}(N)=0$ and $K \leq_{c} N$, then $Z_{\mu M}(N / K)=0$.

Proof. Clear by definitions. 
From now on we consider the condition that for every $M$-singular module $N$, $\operatorname{Rej}(N, \mu)=N$ and give a characterization of GCO-modules and semisimple Artinian rings by considering the classes $\mathcal{M}, \delta$ and $\mathcal{F C}$.

Theorem 3.1. Let $M$ be a module. Consider the following conditions.

a) Every $\mu$-module is projective in $\sigma[M]$.

b) For every $M$-singular module $N, \operatorname{Rej}(N, \mu)=N$.

c) For every $\mu-M$-singular module $N, \operatorname{Rej}(N, \mu)=N$.

d) For every simple $M$-singular module $N, \operatorname{Rej}(N, \mu)=N$.

Then $(a) \Rightarrow(b) \Leftrightarrow(c) \Rightarrow(d)$. If $\mu$ is closed under factor modules, then $(a)-(d)$ are equivalent.

Proof. $(\mathrm{a}) \Rightarrow$ (b) Let $N$ be an $M$-singular module. Let $g: N \rightarrow L$ where $L \in \mu$. Then $N / k e r g \in \mu$. By (a), $N / k e r g$ is projective in $\sigma[M]$. Since $N$ is $M$-singular, we have that $N=k e r g$. Hence $\operatorname{Rej}(N, \mu)=N$.

(b) $\Rightarrow$ (c) Let $N$ be a $\mu-M$-singular module and $g: N \rightarrow L$ a homomorphism where $L \in \mu$. Then $N / k e r g \in \mu$. This implies that $\operatorname{Rej}(N / k e r g, \mu)=0$. Since $N / k e r g$ is $\mu-M$-singular and a $\mu$-module, it is $M$-singular. Then by (b), $N=k e r g$. Hence $\operatorname{Rej}(N, \mu)=N$.

$(\mathrm{c}) \Rightarrow(\mathrm{b})$ and $(\mathrm{b}) \Rightarrow(\mathrm{d})$ are clear.

(d) $\Rightarrow$ (a) Assume that $\mu$ is closed under factor modules. Let $N \in \sigma[M]$ be a $\mu-$ module. We claim that $N$ is semisimple. Let $x \in N$ and $K$ be a maximal submodule of $x R$. Then $x R / K$ is a simple $\mu$-module. By (d) it cannot be $M$-singular. Hence $x R / K$ is projective in $\sigma[M]$. This implies that $K$ is a direct summand of $x R$. Hence $N$ is semisimple. Because of the above process, any simple submodule of $N$ is projective in $\sigma[M]$. It follows that $N$ is projective in $\sigma[M]$.

If we consider the class $\mathcal{M}$ of all $M$-small modules we have a characterization of GCO-modules: A module $M$ is called a $G C O-$ module if every simple $M$-singular module is injective in $\sigma[M]$. $\left(\mathrm{see}^{1}\right)$.

Corollary 3.1. Let $M$ be a module. Then the following are equivalent.

a) Every $M$-small module is projective in $\sigma[M]$.

b) Every $M$-singular module is non-M-cosingular.

c) Every $\mathcal{M}-M$-singular module is non-M-cosingular.

d) $M$ is a GCO-module.

e) Every $\delta-M$-singular module is non-M-cosingular.

Proof. $(d) \Leftrightarrow(a)$ is $b^{7}$ and $(b) \Leftrightarrow(d)$ is by. ${ }^{10}$

Simple modules are either $M$-injective or $M$-small. Hence (a)-(d) are equivalent by Theorem 3.1. (e) $\Rightarrow(b)$ is clear. Since $\mathcal{M} \subseteq \delta$, every $\delta$ - $M$-singular module is $\mathcal{M}-M$-singular. Hence $(\mathrm{c}) \Rightarrow(e)$ is clear.

For the class $\delta$ of all $M$-cosingular modules, we immediately have the following corollary. The equivalencies of (a), (b) and (d) are given in. ${ }^{10}$ 
Corollary 3.2. Let $M$ be a module. Consider the following conditions.

a) Every $M$-cosingular module is projective in $\sigma[M]$.

b) For every $M$-singular module $N, \operatorname{Rej}(N, \delta)=N$.

c) For every $\delta-M$-singular module $N, \operatorname{Rej}(N, \delta)=N$.

d) $M$ is a GCO-module.

Then $(a) \Rightarrow(b) \Leftrightarrow(c) \Rightarrow(d)$. If $\delta$ is closed under factor modules (see Theorem 2.1), then (a)-(d) are all equivalent.

Talebi and Vanaja ${ }^{14}$ are also studied the modules $M$ such that every $M-$ cosingular module is projective in $\sigma[M]$.

A module $M$ is called finitely cogenerated if $\operatorname{Soc}(M)$ is finitely generated and essential submodule of $M$. Let $\mathcal{F C}$ be the class of all finitely cogenerated $R$-modules. Note that $\mathcal{F C}$ is closed under submodules.

Corollary 3.3. The following are equivalent for a ring $R$.

a) Every finitely cogenerated $R$-module is projective.

b) For every singular $R$-module $N, \operatorname{Rej}(N, \mathcal{F} C)=N$.

c) For every $\mathcal{F C}$-singular $R$-module $N, \operatorname{Rej}(N, \mathcal{F} C)=N$.

d) $R$ is semisimple Artinian.

Proof. (a) $\Rightarrow$ (b) $\Leftrightarrow$ (c) By Theorem 3.1.

(d) $\Rightarrow$ (a) is clear.

(b) $\Rightarrow$ (d) Let $E$ be an essential right ideal of $R$. Suppose that $a$ is an element of $R$ but $a$ does not belong to $E$. Let $F$ be a right ideal of $R$ maximal with respect to the properties that $E$ is contained in $F$ and $a$ does not belong to $F$. Then $(a R+F) / F$ is simple singular. By (b), we have a contradiction. Hence $R$ is semisimple Artinial

A ring $R$ is a quasi-Frobenius ring (briefly QF-ring) if and only if every right $R$-module is a direct sum of an injective module and a singular module. ${ }^{11}$ In this result we may take $\mu$-singular modules instead of singular as the following result shows.

Theorem 3.2. The following are equivalent for a ring $R$.

a) $R$ is a $Q F-$ ring.

b) Every right $R$-module is a direct sum of an injective module and a $\mu$-singular module.

Proof. (a) $\Rightarrow$ (b) It is clear.

(b) $\Rightarrow$ (a) Let $M$ be a projective $R$-module. Then $M$ is a direct sum of an injective module and a $\mu$-singular module. Since projective $\mu$-singular modules are zero, $M$ is injective. Then $R$ is a $\mathrm{QF}-$ ring (see for example ${ }^{1}$ ).

Acknowledgment. The author is supported by the project of Hacettepe University of number $05 \mathrm{G} 602$ 001. The referee deserves thanks for careful reading and useful comments. 


\section{References}

1. N.V. Dung, D.V. Huynh, P.F. Smith, R. Wisbauer, Extending Modules, Pitman RN Mathematics 313, Longman, Harlow, 1994.

2. K.R. Goodearl, Ring Theory, Marcel-Dekker, 1976.

3. S.H. Mohamed and B.J. Müller, Continuous and discrete modules, London Math.Soc. LN.147, Cambridge University Press, NewYork Sydney, 1990.

4. K. Oshiro, Lifting modules, extending modules and their applications to QF-rings, Hokkaido Math. J. 13 (1984) 310-338.

5. B. Osofsky, On twisted polynomial rings, J. Algebra 18 (1971) 597-607.

6. A.Ç. Özcan, Some characterizations of V-modules and rings, Vietnam J.Math., 26(3) (1998) 253-258.

7. A.Ç. Özcan, On GCO-modules and $M$-small modules, Comm. Fac. Sci. Univ. Ank. Series A1, 51(2) (2002), 25-36.

8. A.Ç. Özcan and P.F. Smith, The $\mathrm{Z}^{*}$ functor for rings whose primitive images are artinian, Comm. Algebra, 30(10) (2002), 4915-4930.

9. A.Ç. Özcan and A. Harmancı, The torsion theory generated by M-small modules, Algebra Coll., 10(1), (2003), 41-52.

10. A.Ç. Özcan, The torsion theory cogenerated by $\delta$ - $M$-small modules and GCOmodules, Comm. Algebra 35, 623-633 (2007).

11. M. Rayar, On small and cosmall modules, Acta Math. Acad. Sci. Hungar., 39(4) (1982) 389-392.

12. M. Rayar, A note on small rings, Acta Math. Hung., 49(3-4) (1987), 381-383.

13. D.W. Sharpe and P. Vamos, Injective Modules, Cambridge University Press: Cambridge, 1972.

14. Y.Talebi, N. Vanaja, The torsion theory cogenerated by M-small modules, Comm. Algebra, 30(3) (2002) 1449-1460.

15. R. Wisbauer, Foundations of Module and Ring Theory, Gordon and Breach, Reading, 1991.

16. Y. Zhou, Relative socle, relative radical, and chain conditions, Math. Japonica, 38(3) (1993) 525-529.

17. Y. Zhou, Generalizations of perfect, semiperfect, and semiregular rings, Algebra Coll., $7(3)$ (2000) 305-318. 